\title{
High velocity impact and armour design
}

\author{
L. Iannucci ${ }^{1}$, D. Pope ${ }^{2}$ \\ ${ }^{1}$ Imperial College, Department of Aeronautic, South Kensington, SW7 2AZ, London, UK. \\ ${ }^{2}$ Defence Science and Technology Laboratory, Physical Sciences Department, Porton Down, Salisbury, Wiltshire, \\ SP4 0JQ, UK.
}

Received 17 September 2010; accepted in revised form 16 January 2011

\begin{abstract}
Improving combat survivability is the most important aspect of military technology. Hence the development of new lightweight armour systems is a key requirement. A large number of new high performance polymer fibres have been developed in recent years, which include Aramid fibres, polyethylene fibres and polypropylene fibres, amongst others, and have been applied to soft armour systems.

To gain a fundamental understanding of which fibre type is the best for a specific application requires the development of techniques which can span all length scales. It has been widely recognised that multiscale modelling, which encompasses the full range of length and time scales, will be an important factor in the future design and testing of novel materials, and their application to armour design.

In the present paper a new material damage model suitable for the simulation of impact on thin laminated panels fabricated from high performance fibres is implemented into the commercial ls-dyna ${ }^{\circledR}$ finite element code. The new material model links the mesoscale behaviour of the individual fibres to the macroscale behaviour within a conventional shell finite element. The implemented model is used in a parametric high velocity study to illustrate the applicability of the model to the design of thin armour panels.
\end{abstract}

Keywords: modeling and simulation, armour design, finite elements

\section{Introduction}

A continued need exists for high performance, lightweight and cost effective protection for personnel and vehicles to improve survivability and reduce injury when subject to a range of potential threats e.g. blast and ballistic impacts. Protection or armour systems can be broadly divided into hard ceramic based systems, with high performance fibres within a composite backing; these are used for both personnel and vehicle armour, since they can be very effective at stopping ballistic projectiles by breaking and eroding them, and soft armour which use high performance composites to primarily prevent fragmentation incursion [1]. The advantages being these systems are very light, e.g. high performance polyethylene has a density less than water.

During the last 15 years a large number of new high performance polymer fibres with aligned carbon chains have been developed, which include Aramid fibres $\left(\right.$ Kevlar $^{\circledR}$, Twaron $\left.{ }^{\circledR}\right)$, polyethylene fibres $\left(\right.$ Dyneema $^{\circledR}$, Spectre $^{\circledR}$ ) and polypropylene fibres $\left(\right.$ Curv $^{\circledR}$, Tegris $\left.^{\circledR}\right)$. Hence a reliable technique is a requirement to enable ranking of potential solutions and to determine the most effective combination of fibres for specific threats.

The classic understanding of how soft ballistic armour functions assumes that the impact energy can be dissipated by waves propagating along the fibres and hence the controlling materials properties

\footnotetext{
${ }^{*}$ Corresponding author, e-mail: 1.iannucci@imperial.ac.uk
} (c) BME-PT 


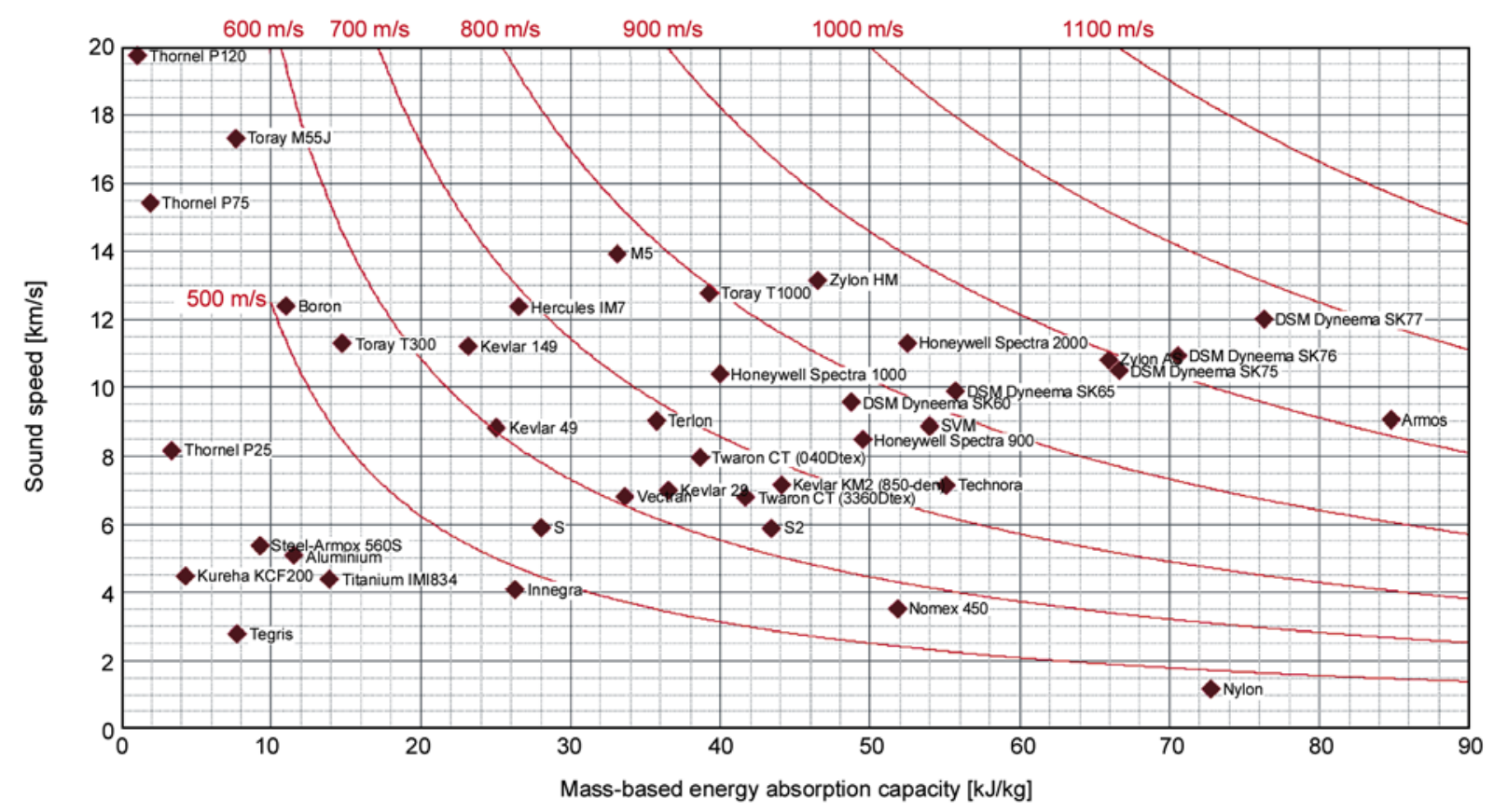

Figure 1. Sound speed vs. mass-based energy absorption capacity for a number of high-performance fibres [2] superimposed with Cunniff's $U^{*}$ velocity [3]

are the tentsile wave velocity in the fibres and the specific energy absorbed at failure, this makes high performance fibres particularly attractive in impact protection systems and containment devices, this is schematically shown with the most common high performance fibres in Figure 1.

The modelling of ballistic impact onto panels composed of high performance fibres can be divided into semi-analytical and numerical based techniques. The most common analytical based technique, which is often used to scale impact and hence determine the ballistic performance of different panel thicknesses was developed by Cunniff [3]. Figure 1 is often used to determine the 'best' fibre for a soft armour system in Cunniff's approach (e.g. the highest dimensionless velocity), however, in this selection the assumption is implicit that the fibres are uniformly loaded; the failure response is linear from the ultimate strength of the fibre to rupture of the fibre with no plasticity assumptions, associated with the low compressive strength and unloading prior to complete failure. The non-linear shear response is also not considered. Despite these drawbacks the approach has found success. However, some exemptions have been noted, for example brittle systems based on carbon and Tegris. The inclusion of rate effects and phase change in the material is also a conceptually a difficult task to incorporate into such an approach. An alternative methodology is to use hydrocodes, e.g. 1s-dyna ${ }^{\circledR}$ or abaqus ${ }^{\circledR}$ which includes the full constitutive relationship. An excellent review of numerical approaches is discussed in Grujicic et al. [4] and includes a multi-scale strategy based on Dyneema $\mathrm{UD}^{\circledR}$. However, the non-linear shear response is also not considered. The importance of this behaviour is further discussed in section 3.0.

It has been widely recognised that the advent of multiscale modelling, which encompasses the full range of length and time scales, will be an important factor in the future design and testing of novel materials [4]. In the present approach the methodology adopted by Cunniff is incorporated within a numerical finite element framework with enhancements associated with the physical behaviour of the material, specifically the non-linear shear and compressive behaviour.

\section{Laboratory tests}

A series of laboratory tests were performed on Dyneema UD composites fabricated from Dyneema UD tape, which has a lamination of 0/90/0/90 and a typically thickness of the order of $\sim 270 \mu \mathrm{m}$. The Dyneema UD composite fabricated from the tape usually has a very low shear and compressive strengths, while the tensile strength and strain to failure are excellent for a polymer. However, the measured composite laminate properties are not 

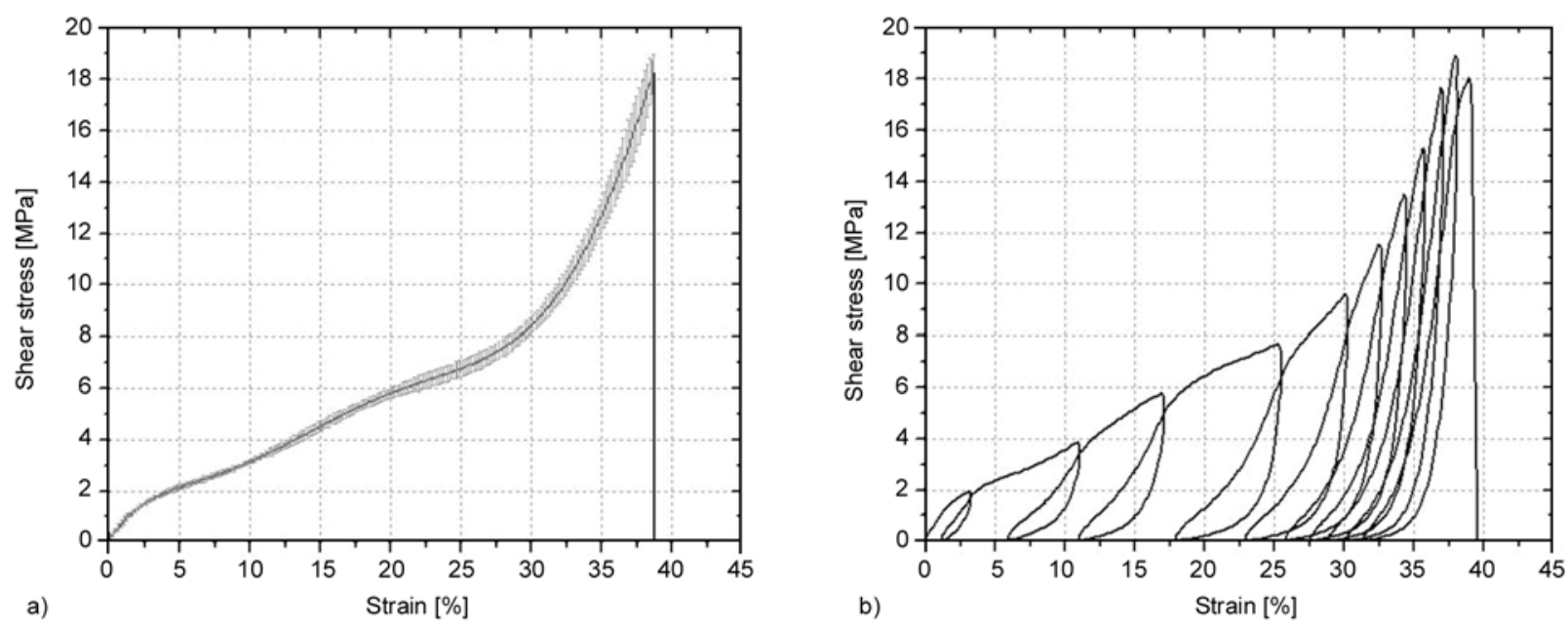

Figure 2. Tension-shear stress strain curve for Dyneema UD composite [5]: a) Increasing load until failure; b) Cyclic load/unload until failure

used in the following model, only tensile fibre stiffness and strength available in the open literature. To investigate the non-linear shear response a series of tests [5] were performed in a standard tension-shear configuration to investigate the behaviour in pure in-plane shear. This is a necessary parameter for the proposed model. These tests were carried out following the BS EN ISO 14129:1998 standard. All tests were performed on specimens with a width of $25 \mathrm{~mm}$, a thickness of $5 \mathrm{~mm}$ and a length of $250 \mathrm{~mm}$ (150 mm gauge length) with a lamination of $\pm 45^{\circ}$ as stated in the standard. A specimen width to thickness ratio $(w / t)$ of 2.5 was selected to ensure that the edge effect was minimal.

Figure 2a illustrates the non-linear shear with the error bars associated with the scatter in three tests. Figure $2 \mathrm{~b}$ illustrates the cyclic unloading behaviour. A highly non-linear behaviour during unloading is observed this is potentially due to matrix damage, viscoplastic effects and the friction between the failed surfaces. This is clearly evident in Figure 3.

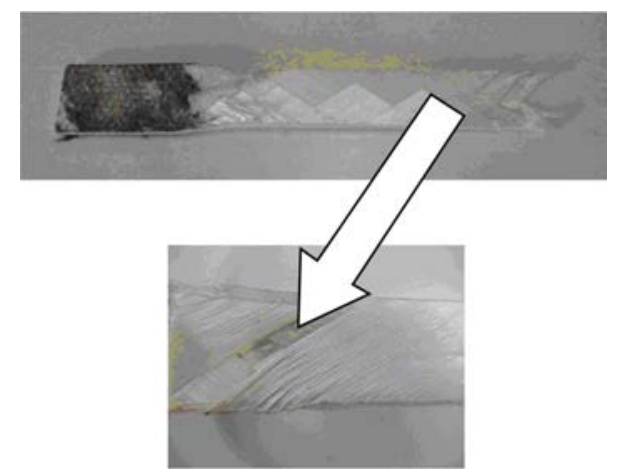

Figure 3. Failed tension-shear specimens (most layers have delaminated as can be seen from the lower figure)
As the fibres re-orient towards the end of the loading regime the stiffening response of the specimen is potentially associated with fibre reorientation.

Failure appeared to initiate from the grips, generating a series of fracture (delaminations and matrix cracking) surfaces along the specimen in a cone shaped pattern. A final series of static tests were performed to investigate the compressive behaviour [5]. In the present study the BS EN ISO 14126:1999 was used for the compressive test. The stress strain relationship is shown in Figure 4. The compressive behaviour is dominated by microscale kinking of the fibres. The initial peak is probably associated with their formation, followed by a plateau regime in which the kinks slide as more or less constant stress. Thus in the following modelling section it is proposed to model the compressive behaviour as an elastic-plastic material along fibre directions only with a typical strength of $8 \mathrm{MPa}$.

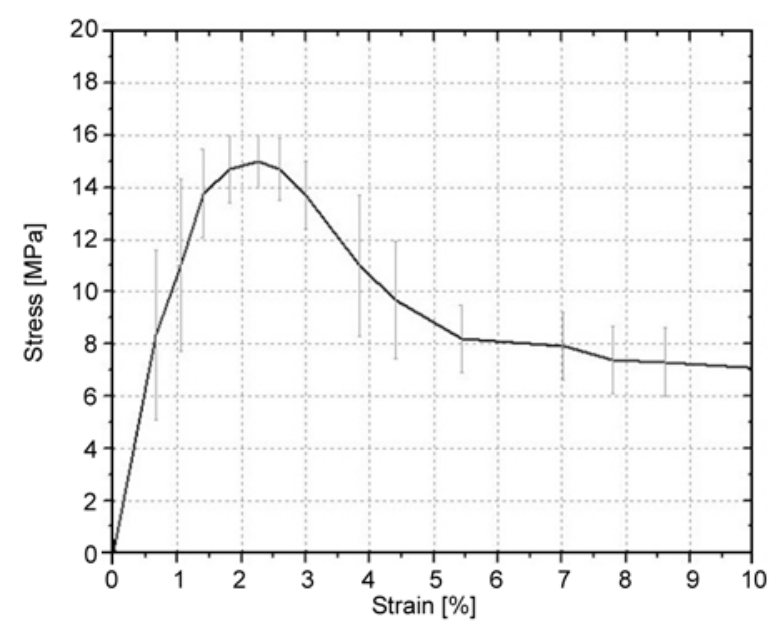

Figure 4. Compression test: stress versus strain relationship 
Table 1. Summary of experimental results from high velocity gas gun testing

\begin{tabular}{|l|c|c|c|c|c|}
\hline \multicolumn{1}{|c|}{ Testing regimes } & Projectile used & $\begin{array}{c}\text { Mass of projectile } \\
{[\mathbf{g}]}\end{array}$ & $\begin{array}{c}\text { Velocity range } \\
{[\mathbf{m} / \mathbf{s}]}\end{array}$ & $\begin{array}{c}\text { Areal density } \\
{\left[\mathbf{k g} / \mathbf{m}^{\mathbf{2}}\right)}\end{array}$ & $\begin{array}{c}\text { Ballistic limit } \\
{[\mathbf{m} / \mathbf{s}]}\end{array}$ \\
\hline $\begin{array}{l}0.8 \mathrm{~mm} \text { Dyneema UD panels } \\
(100 \mathrm{~mm} \times 100 \mathrm{~mm})\end{array}$ & $6 \mathrm{~mm}$ Mild Steel ball & 0.8677 & $305-388$ & 5.7 & 305 \\
\hline
\end{tabular}

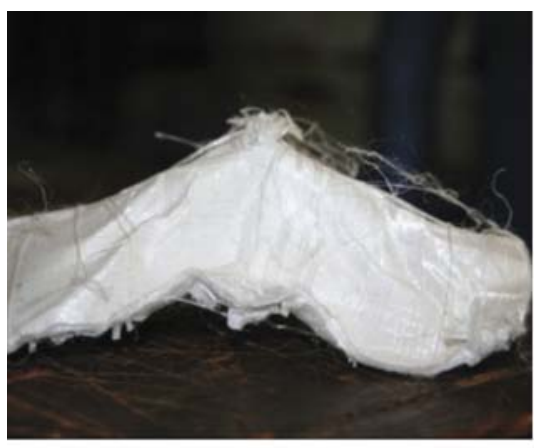

a)

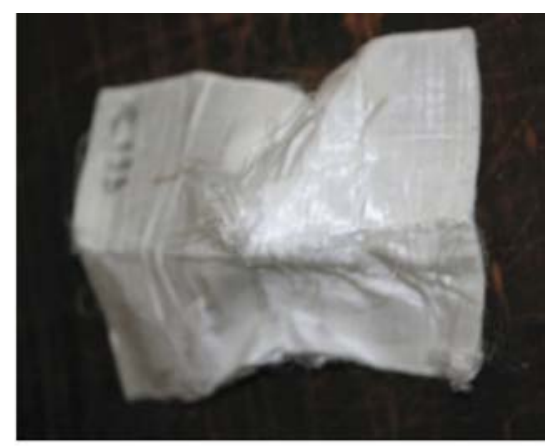

b)

Figure 5. Ballistic impact on Dyneema UD crossply laminate near the ballistic limit: a) Side view of specimen; b) Rear view of specimen

A series of impacts were also performed on Dyneema UD crossply laminates [5] to determine the ballistic limit and to use the results to validate the proposed constitutive model, Table 1. The Dyneema samples were mounted on a frame, with lightly clamped edges, which allowed the material to draw, i.e. no bolts or rigid clamps were used. Figures $5 \mathrm{a}$ and $5 \mathrm{~b}$ show the panel near the ballistic limit. The excessive displacements and drawing of the laminate is clearly evident. The deformation pattern would indicate a very low shear and compressive strengths. This has been confirmed from the static test conducted.

\section{Model development}

An elegant analytically based procedure was developed by Cunniff [3] to determine the $\mathrm{V}_{50}$ of an armour system. The key properties proposed by Cunniff for an armour system were;

the presented area and mass for the projectile;

the fibre sonic wave speed;

the fibre fracture toughness for the fibre.

The dimensionless ratios for the system and defined by Cunniff were:

$\sigma \quad-$ Fibre ultimate axial tensile strength

$\varepsilon \quad-$ Fibre ultimate tensile strain

$\rho \quad-$ Fibre density

$E \quad-$ Fibre modulus (assumed linearly elastic)

$A_{\mathrm{p}}$ - Projectile presented area

$A_{\mathrm{d}} \quad-$ System areal density

$m_{\mathrm{p}} \quad$ - Projectile mass
$\mathrm{V}_{50}-\mathrm{V}_{50}$ ballistic limit (the velocity at which impacting projectiles are expected to defeat a system $50 \%$ of the time)

$U^{*} \quad$ - The product of fibre specific toughness and fibre strain wave velocity.

This leads to the Equations (1) and (2), which can be used to determine the $V_{50}$ of an armour system, if the fibre type is known.

$\Phi=\left(\frac{\mathrm{V}_{50}}{\left(U^{*}\right)^{1 / 3}} \mid \frac{A_{\mathrm{d}} A_{\mathrm{p}}}{m_{\mathrm{p}}}\right)$

where

$U^{*}=\left(\frac{\sigma \varepsilon}{2 \rho}\right) \sqrt{\frac{E}{\rho}}$

the product of the fibre specific toughness and fibre strain wave velocity.

Cunniff developed the so called ' $U^{*}$ ' parameter to predict ballistic impact resistance in fibres. The model has been generally successful in predicting performance for a wide range of fibre/textile materials.

This paper describes the use of an existing model developed for low velocity impacts, which was implemented into the explicit FE code LS-DYNA, however, a number of key modification were necessary to allow for a realistic behaviour [6]. The formulation is concerned primarily with in-plane failure of thin high performance laminated UD composites. In the present paper it is compared with experimental results for a thin Dyneema UD com- 
posite, however, it can be used for any high performance system. The inherent assumption follows the bilinear assumption in Cunniff paper, but includes plasticity and a non-linear shear response, which are both important. In the present formulation two damage variables are introduced to model the observed fibre damage. Compression failure is model with two plastic strain measures. The shear response follows a non-linear behaviour. Unloading always follows the elastic unloading slope before damage commences, but unloads with the damaged modulus when damage has initiated. Again, this is a convenient assumption, which captures the non-linear elastic and permanent strain behaviour. The fracture process is implicit within the stress-strain relationship for the composite material. The damage mechanics methodology must hence be based on a Unit Cell (UC), unlike classical fracture mechanics.

\subsection{Stiffness degradation}

Each Unit Cell (UC) consists of a 0 and 90 UD high performance ply; basically a cross-ply. Using a simple mosaic modelling approach it is possible to derive the cross-ply stiffness in each local direction within the unit cell, i.e. 0 and 90 layer.

Figure 6 illustrate a typical Dyneema cross-ply layer with the 0 degree layer removed. The ridges relate to the resin tracks. Note the low apparent resin; this is typical for a composite system in which the volume fracture of resin may be $\sim 15 \%$. Hence for the local $x$ and $y$-fibre directions and assuming the fibre stiffness dominates the ply stiffness: The modulus and its degradation can be expressed by Equations (3) and (4):

$E_{11}=0.5\left(V_{\mathrm{f}} E_{\mathrm{f}}\right)\left(1-d_{1}\right)$

$E_{22}=0.5\left(V_{\mathrm{f}} E_{\mathrm{f}}\right)\left(1-d_{2}\right)$

with the following definitions;

$d_{1}=$ tensile failure in $x$ direction fibre,

$d_{2}=$ tensile failure in $y$ direction fibre,

$E_{\mathrm{f}}=$ Young's Modulus of fibre,

$V_{\mathrm{f}}=$ Volume fracture of fibre.

The damage parameters relate to the degradation of the fibres in the 0 and 90 layers within the unit cell in tension only. The fibre modulus is assumed constant and has been taken from the open literature [8]. The introduction of rate and temperature effects

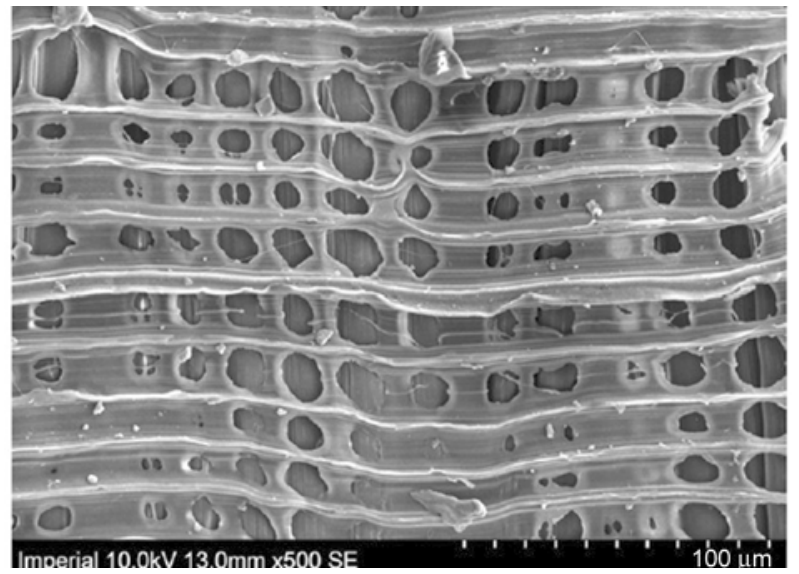

Figure 6. Interlaminar voids in Dyneema UD composites [7]

[11-13] into the model can also be achieved, however, a relationship between rate and temperature needs to be developed as does a technique to determine adiabatic heating effects, and the corresponding change in stiffness, strength and fracture energy.

A simple non-linear shear stress-strain behaviour is assumed for the in-plane shear response. The shear stress-strain response is defined by Equation (5):

$\tau=A \gamma^{3}+B \gamma^{2}+C \gamma+D$

where $D=0.0, C=109.61, B=-580.424, A=$ 1120.77 in units of MPa for the curve shown in Figure 2a. A simple least square fit was used to derive the constants from the experimental curve. The instantaneous shear modulus can be trivially derived using the above equation. Since the modelling approach uses shell element only, with no delamination between plies. The non-shear shear response must include all possible shear failure modes; hence degradation associated with delaminations must be included implicitly within the response. These delaminations can be clearly seen in Figure 3, which results in the curve shown in Figure $2 \mathrm{a}$, and the progression of damage in Figure $2 \mathrm{~b}$.

\subsection{Fibre fracture evolution}

Failure in both the 0 and 90 directions is formulated in a similar manner. No cross coupling between 0 and 90 failure is included within the constitutive model. Once the initiation (failure) stress is reached damage initiates and stress is gradually reset to zero in either the 0 or 90 direction as damage reaches a value of one. Therefore element deletion could be used to represent a physical tear in the laminate, if 


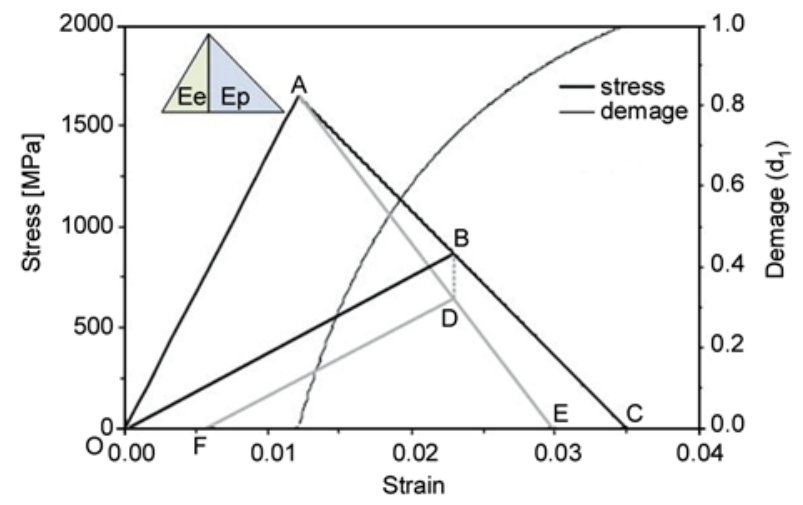

Figure 7. Assume stress-strain response for high performance fibre. OAE relates to a response with permanent strain, unloading would then follow OADF [6]

damage reaches one in either the 0 or 90 directions for all integration points within the element (i.e. all laminae layers have failed). For the 0 and 90 fibre fracture case $(i=1,2)$, the damage evolution equation is defined as, OAC in Figure 7, when no permanent strain is present, and unloading between BO for any damage $d$ (Equation (6)):

$d_{\mathrm{i}}=\frac{\varepsilon_{\max , \mathrm{i}}}{\left(\varepsilon_{\max , \mathrm{i}}-\varepsilon_{0, \mathrm{i}}\right)}\left[1-\frac{\varepsilon_{0, \mathrm{i}}}{\varepsilon_{\mathrm{ii}}}\right]$

where $\varepsilon_{\text {max }, \mathrm{i}}$ is the strain at zero stress and damage $=1$, and $\varepsilon_{0}$ is the strain at maximum stress (failure stress) and damage $=0$. The only parameters required for this evolution model are these two strain constants, which define the total energy dissipated, i.e. the area under the stress-strain curve. Equation (5) can be converted into an incremental form, which has been implemented into the LSDYNA code and is stated by Equation (7):

$\Delta d_{\mathrm{i}}=\frac{\varepsilon_{\max , \mathrm{i}}}{\left(\varepsilon_{\max , \mathrm{i}}-\varepsilon_{0, \mathrm{i}}\right)}\left[\frac{\varepsilon_{0, \mathrm{i}}}{\varepsilon_{\mathrm{ii}}^{2}}\right] \Delta \varepsilon_{\mathrm{ii}}$

The constants, $\varepsilon_{\max , \mathrm{i}}$ and $\varepsilon_{0, \mathrm{i}}$ must be chosen to prevent a mesh dependent solution by maintaining constant energy dissipation independent of volume, which corresponds to a physical element size, i.e. $\varepsilon_{\text {max,i }}$ must be altered at an element level. This is easily input as a parameter at an element level, or calculated within the element subroutine based on the element volume. The damage evolution can then be trivially stated as Equation (8):

$d_{\mathrm{i}}^{\mathrm{n}+1}=d_{\mathrm{i}}^{\mathrm{n}}+\Delta d_{\mathrm{i}}^{\mathrm{n}+1}$

where ' $n$ ' represents the $n^{\text {th }}$ timestep or load increment.
Examination of Figure 7 reveals that the basic shape follows the assumptions in Cunniff's model. Clearly to include plasticity it is necessary to offset the unloading curve by an appropriate amount so that it unloads prior to the origin, i.e. DF. The offset $\mathrm{BD}$ relates to an irreversible damage constant which is described in the next section.

\subsection{General plane stress stress-strain-damage relationship}

The general plane stress stress-strain relationship for the damage model can be derived directly from Hooke's law. A general form could be described by Equation (9) and can be expanded into incremental form to include a permanent or damage strain component. The magnitude of the permanent damage strain can be determined via the material constant $\beta$.

$\dot{\sigma}=C \dot{\varepsilon}+\beta \dot{C} \varepsilon$

The permanent damage strain would result in a permanent fix if unloading occurs prior to failure. Hence to determine the $\beta$ constants it is necessary to perform such tests, unfortunately such dynamic tests would be very difficult to achieve and static unloading may be the only appropriate test.

Cross-coupling and interaction terms are not considered in the present formulation [6]. The stressstrain-damage relationship is hence defined by Equations (10) and (11):

$\dot{\sigma}=C \dot{\varepsilon}+\dot{\sigma}_{\text {ir }}$

where

$\dot{\sigma}_{\mathrm{ir}}=\left(\begin{array}{c}-\beta_{1} \sigma_{11} \frac{\dot{d}_{1}}{\left(1-d_{1}\right)} \\ -\beta_{2} \sigma_{22} \frac{\dot{d}_{2}}{\left(1-d_{2}\right)} \\ 0\end{array}\right)$

The $\beta_{\mathrm{i}}$ terms in the above equation control the amount of residual permanent strain (plastic strain). Consider the unloading point $\mathrm{B}$ in Figure 7; with $\beta_{\mathrm{i}}=1.0$ the unloading path is directly to the origin with no residual plastic strain, while a value of $\beta_{\mathrm{i}}>1.0$ result in a positive residual plastic strain, i.e. path BDF, as the strain softening line $\mathrm{AC}$ has now moved to a position $\mathrm{AE}$ to accommodate the additional stress reduction. A value of $\beta_{\mathrm{i}}<1.0$ is not permitted, as this would indicate an unrealistic neg- 
ative permanent strain. In the present formulation for the irreversible stress, $\dot{\sigma}_{\mathrm{ir}}$, second order terms are neglected.

\subsection{Work dissipated}

Work dissipation is included in the implementation for information only and is not directly used within the stress update procedure. The work dissipated $\dot{W}_{\mathrm{i}}$ for a damage rate $\dot{d}_{\mathrm{i}}$ is given by Equation (12) [6]:

$\dot{W}_{\mathrm{i}}^{\mathrm{n}}=\frac{\left(2 \beta_{\mathrm{i}}-1\right)}{2} \frac{\sigma_{\mathrm{ii}}^{2}}{E_{\mathrm{ii}}^{0}\left(1-d_{\mathrm{i}}\right)^{2}} \dot{d}_{\mathrm{i}}^{\mathrm{n}}$

where ' $n$ ' denotes the $n^{\text {th }}$ time step or load increment, and $E_{\mathrm{ii}}^{0}$ relates to the undamaged modulus.

Clearly the total energy dissipated can be predicted for a specific volume of material. This can be used to understand which regions are dissipating the most energy, or power. Hence different fibres could be used in different layers depending on the energy dissipated.

\subsection{Permanent plastic strain}

The total strain is the sum of permanent (plastic) and elastic strain. From the stress-strain curve, it can be shown that the plastic strain (OF) in the tensile case, Figure 7, is given by Equation (13) [6]:

$$
\dot{\varepsilon}_{\mathrm{pl}, \mathrm{i}}=\left(\beta_{\mathrm{i}}-1\right) \frac{\sigma_{\mathrm{ii}}}{E_{\mathrm{ii}}^{0}\left(1-d_{\mathrm{i}}\right)^{2}} \dot{d}_{\mathrm{i}}
$$

the cumulative permanent strain is trivially defined by Equation (14):

$\varepsilon_{\mathrm{pl}, \mathrm{i}}^{\mathrm{n}+1}=\varepsilon_{\mathrm{pl}, \mathrm{i}}^{\mathrm{n}}+\Delta \varepsilon_{\mathrm{pl}, \mathrm{i}}^{\mathrm{n}+1}$

where ' $n$ ' represents the $n^{\text {th }}$ timestep or load increment. Figure 7 illustrates the bilinear constitutive model where $\mathrm{AC}$ relates to $\beta_{\mathrm{i}}=1$ and $\mathrm{AE}$ when $\beta_{\mathrm{i}}>1$. The greater the value of $\beta_{\mathrm{i}}$, the greater the magnitude of the irreversible stress $\mathrm{BD}$, and hence the permanent strain OF. The 'plastic strain' that is defined in this paper results from incomplete closure of cracks and potential fibre damage formed during damage evolution. It is clear that the $\beta_{\mathrm{i}}$ constants can be derived from experimental cyclic permanent strain versus damage plots.

\subsection{Damage evolution for compressive direct stresses}

In the present formulation, compression failure is modelled in a simple elastic perfectly plastic man- ner. The local $x$ and $y$ fibres have their own plastic strain component and are completely uncoupled. Figure 6 would indicate that this type of modelling approach seems appropriate due to the lack of resin. The fibres would tend to kink, but not fail. The relevant equations for compression for the local $x$ and $y$ fibre directions are given in Equations (15) and (16). No failure strain is applied.

$\mathrm{d} \varepsilon_{\mathrm{i}}=\mathrm{d} \varepsilon_{\mathrm{i}}^{\mathrm{e}}+\mathrm{d} \varepsilon_{\mathrm{i}}^{\mathrm{p}}, \quad f=\sigma_{\mathrm{i}}-\sigma_{\mathrm{y}}$

$\mathrm{d} \varepsilon_{\mathrm{i}}^{\mathrm{p}}=\mathrm{d} \lambda \frac{\partial f}{\partial \sigma_{\mathrm{i}}}$

where $\mathrm{d} \lambda$ is a proportional constant to maintain stresses on the compressive yield surface.

\subsection{Smearing methodology}

The modelling of tearing and fracturing in brittle materials is an inherently mesh dependent problem and was initially encountered, and solutions proposed in the Civil Engineering community [6].

The formulations described in the previous sections relate the energy dissipated within a unit volume to the fracture energy. Hence, it is necessary to relate this energy to the energy required to increase the fracture area or cracked surface. Since Finite Elements are volume based, it is convenient to link damage mechanics and fracture mechanics. Consider the bilinear implementation shown in Figure 7 (insert). The specific energy dissipated $E_{\mathrm{f}}$ can be subdivided into the elastic energy $E_{\mathrm{e}}$ and the propagation energy $E_{\mathrm{p}}$. This leads to the following Equation (17):

$E_{\mathrm{f}}=E_{\mathrm{e}}+E_{\mathrm{p}}$

The energy dissipated should be independent of mesh (finite element) size. If the specific internal energy is constant, the adjusted value of $E_{\mathrm{p}}$ can be determined for different mesh densities (finite element mesh size). For a bi-linear stress-strain-damage curve the specific energy can be defined by Equation (18):

$E_{\mathrm{f}}=\frac{1}{2} \sigma_{0} \varepsilon_{\max }$

and for the individual energies within the bi-linear stress-strain relationship, Equation (19):

$E_{\mathrm{p}}=\frac{1}{2} \sigma_{0}\left(\varepsilon_{\max }-\varepsilon_{0}\right)$ and $E_{\mathrm{e}}=\frac{1}{2} \sigma_{0} \varepsilon_{0}$ 
This intralaminar fracture energy, which is a fracture mechanics based quantity, can be related to the energy dissipated within the finite element volume by linked damage and fracture mechanics. Equation (19) illustrates the energy dissipated for a particular damage mode, $\Pi$, and the coupling between damage and fracture mechanics leads to Equation (20):

$\prod=\left(E_{\mathrm{e}}+E_{\mathrm{p}}\right) A l_{\mathrm{x}}=G_{\mathrm{f}} A$ and $l_{\mathrm{x}}=\frac{A_{\mathrm{e}}}{l}$

where

$A_{\mathrm{e}}=$ shell area (mesh parameter),

$A=$ fracture area (mesh parameter),

$G_{\mathrm{f}}=$ intralaminar fracture energy (material property),

$l=$ longest length (mesh parameter),

$l_{\mathrm{x}}=$ characteristic length (mesh parameter).

No minimum element size restriction applies when using Equation (20), except it is desirable that the element size should be equal or greater than the process zone in a particular damage mode, however, a restriction does exist for the maximum element size used in the mesh. Re-arranging Equation (20) and using Equation (19) yields the following inequality, defined by Equation(21):

$l_{\mathrm{x}}<\frac{2 G_{\mathrm{f}}}{\sigma_{0} \varepsilon_{0}}$

This inequality prevents a snap through stress-strain behaviour, i.e. a negative $E_{\mathrm{p}}$. The issues associated with the determination of the failure strain $\varepsilon_{\max }$ are discussed in a later section.

\section{High velocity impact model}

The Unit Cell (UC) represents the behaviour of a cross ply composite. Hence its implementation into a shell element required each integration point to be associated with the UC. For example, if 100 0/90 cross ply are used in the laminate, then 100 integration point should be used to model the macroscale behaviour from the UC mesoscale model. In the present example the experimental results presented in Table 1 are simulated. However, to understand the behaviour of the model for different materials a parameter study is performed with the properties in Table 2. The fracture toughness per unit mass was assumed the same for all high performance fibres listed. The strain at fracture is assumed for Kevlar and Nylon to make the fracture toughness the same
Table 2. High Performance fibre properties used in study

\begin{tabular}{|l|c|c|c|c|}
\hline Fibre type & $\begin{array}{c}\text { Density } \\
{\left[\mathbf{g} / \mathbf{c m}^{\mathbf{3}} \mathbf{]}\right.}\end{array}$ & $\begin{array}{c}\text { UTS } \\
{[\mathbf{G P a}]}\end{array}$ & $\begin{array}{c}\text { Strain at fracture } \\
{[\mathbf{\%}]}\end{array}$ & $\begin{array}{c}\mathbf{E} \\
{[\mathbf{G P a}]}\end{array}$ \\
\hline Nylon & 1.12 & 0.90 & 14.6 & 9.6 \\
\hline Kevlar & 1.44 & 2.50 & 6.8 & 71.0 \\
\hline Dyneema & 0.97 & 3.00 & 3.8 & 116.0 \\
\hline
\end{tabular}

for all composites. This is a simple assumption to illustrate the applicability of the model. The nonlinear shear and compressive behaviour are assumed the same and only the initial stiffness and UTS are altered. This assumption assumes the resin properties and failure would control the shear and compressive response. For a very low resin system this would probably be the case.

\subsection{High velocity impacts on Dyneema UD}

The laboratory impacts described in Section 2. are modelled using the techniques described in section 3 . The projectile is modelled with an elastic plastic material model and the specimen modelled with the new material model. Contact logic is applied at all interfaces between the test rig and the composite and the projectile and the composite. The predicted deformed pattern exhibits the key feature, such as the excessive drawing of the material and the very large displacement prior to perforation.

Figure 8 indicates the behaviour well above the ballistic limit. Element deletion occurs when either the 0 and 90 laminae within the UC has failed. Clearly modelling the tensile, compressive and shear behaviour was key in simulating the correct response. If the impact velocity is reduced towards the ballistic limit the behaviour follows Figure 9. This clearly appears to give the same drawing pattern as the test. The ballistic limit was also approximately the same.

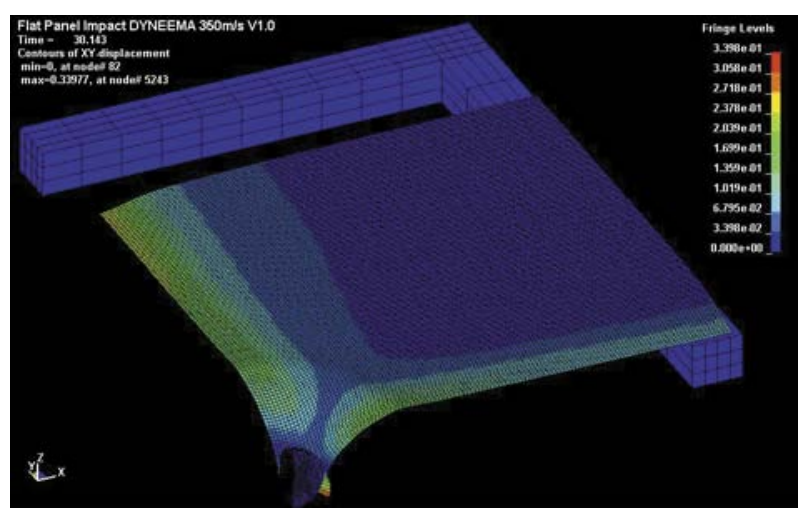

Figure 8. Ballistic impact ( $350 \mathrm{~m} / \mathrm{s}$ above ballistic limit) using Dyneema laminate. XY displacement plot. 


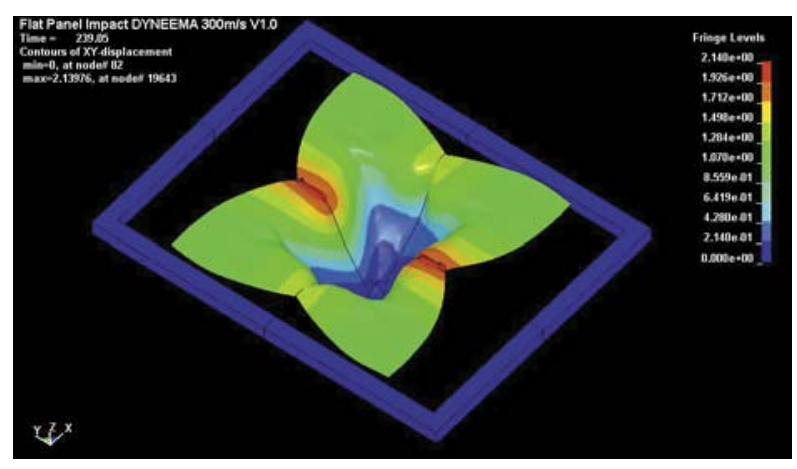

Figure 9. Ballistic impact (300 m/s at ballistic limit) using Dyneema laminate. XY displacement plot.

However, the stiffness and strength are based on the fibre values only!

\subsection{High velocity impacts on other high performance UD materials}

The model is applied to a hypothetical Kevlar material with realistic fibre stiffness and strength. The procedure used for the Dyneema is repeated and the ballistic limit determined. The velocity at $350 \mathrm{~m} / \mathrm{s}$ is shown in Figure 10. The final ballistic limit was found to be between $200-250 \mathrm{~m} / \mathrm{s}$, considerably lower than the Dyneema.

To confirm the behaviour was realistic the analyses was repeated for Nylon. The behaviour is shown in Figure 11. Clearly the drawing behaviour is different; however, the same energy and shear behaviour were used. This would indicate that the fibre stiffness and strength are the important parameters and confirms Cunniff original assumptions. The ballistic limit was again considerably below that of Dyneema.

High performance fibre test results are generally available in the open literature, for example in [8, 9]. These tests are normally conducted on fibres

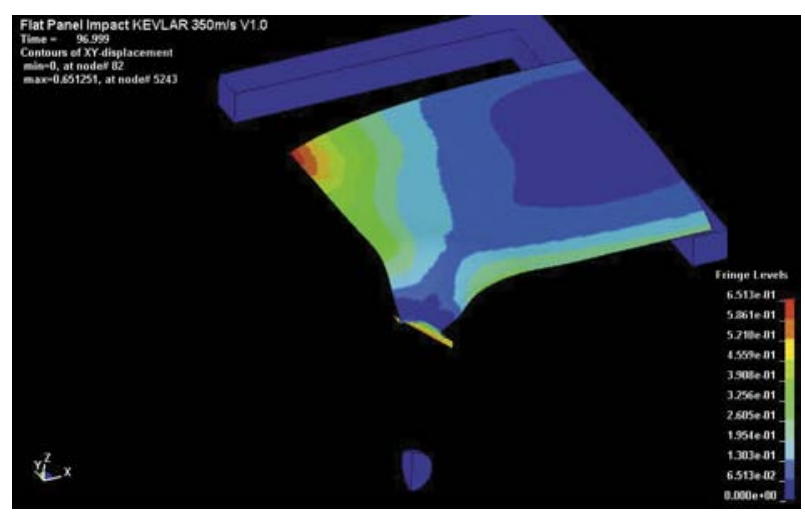

Figure 10. Ballistic impact ( $350 \mathrm{~m} / \mathrm{s}$ above ballistic limit) using Kevlar laminate. XY displacement plot.

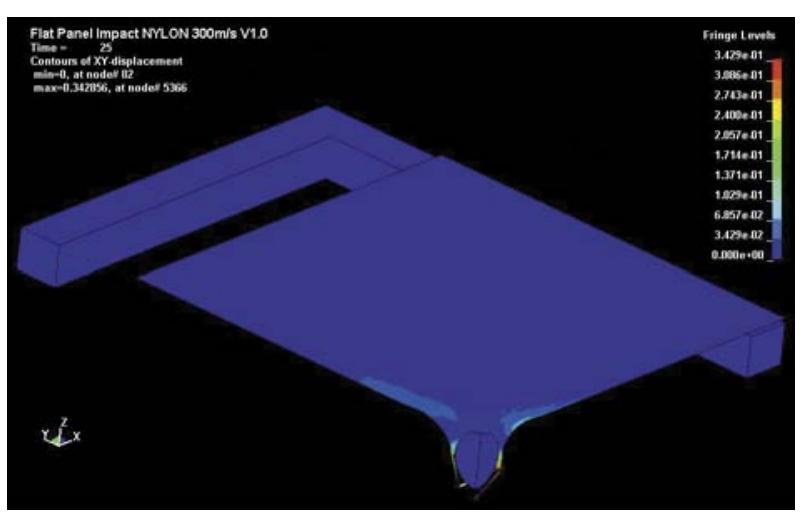

Figure 11. Ballistic impact (300 m/s above ballistic limit) using Nylon laminate. XY displacement plot.

with yarns, typically 300 fibres or greater, hence the stress in calculated based on the number and crosssection area of these fibres. The strain is usually determined via a contactless video device between two known gauge points, perhaps several centimetres apart, while for the dynamic tests several millimetres, a requirement for the dynamic tests to achieve equilibrium. Examination of the failure mechanisms clearly indicates a weakest link type failure with individual fibres failing within the gauge length, thus generating a fluffy appearance. Thus no clear fracture surface is evident, which is what would normally be expected for carbon and glass based systems using a brittle matrix. Thus, how is the strain at fracture, which could be between $\mathrm{A}$ and $\mathrm{E}$ in Figure 7, determined for these materials? For brittle composite systems it can be relatively easily determined by performing additional fracture mechanics type tests [10] to determine the fracture energy, and if the strength is known the ultimate fracture strain can be determined for the material model. For fibre tests, a variation in the failure stress is observed and is generally a function of the number fibres in the yarn, and the gauge length [9]. This is usually modelled via a Weibull type approach on the strength, however, details on the variation of the failure strain and hence total fracture energy absorbed is not available in the open literature. This does pose the problem whether a probabilistic type approach should be adopted since each Finite Element has a finite volume, hence with the use of a Weibull distribution and Monte-Carlo type simulations such an approach could be adopted. This poses the question on which representative volume should be used for such an analysis. Returning to the original question 
on how to select the appropriate failure strain this should be taken from Hopkinson bar tests for the anticipated strain rates. The static test results cannot generally be used for reasons associated with strainrate, visco-elastic effects, etc. but more importantly since the tests are conducted at static strain rates once localisation and failure occurs in individual fibres, elastic strain energy stored in the remaining broken fibres elastically unload almost immediately causing an abrupt unloading on the measured gauge displacement. This unloading process is restricted in the dynamics test, since at the higher strain rates the unloading wave take a finite time to relaxes the fibre, thus measuring the familiar triangular or half sine wave shaped stress-strain curve as the force is recovered from the bars, not directly at the specimen. This is well illustrated in [9].

\section{Conclusions}

The modelling approach appears to confirm the originally assumptions developed by Cunniff in his paper. The advantage of using numerical techniques is that it allows the contact logic inherent within most Finite Element codes to model different projectiles and their behaviour. Thus projectile area and shape are modelled explicitly.

The approach can also be used to understand the observed deviations of polypropylene performance from the established correlations to Cunniff original formulations. However, a robust methodology needs to be developed to determine the final failure strain to be input into the material model. Similarly to determine the residual plastic strain cyclic unloading tests would be required. Furthermore, the approach can be applied to the development of hybrid layers within an armour system. This could follow the natural process often found in nature, e.g. spider silks [14].

To model the actual hydrodynamic event at the point of impact the EOS must be included within the modelling approach [15]. As the model is presented it is most suitable for the rear part of a soft armour system were hydrodynamic effects are substantial smaller. Modelling the complete penetration including all damage mechanisms remains conceptually a difficult task with normal 3D Finite Element [16].

\section{Acknowledgements}

The support from the Dstl SAMPLE programme gratefully acknowledged.

\section{References}

[1] Jacobs M. J. N., Van Dingenen J. L. J.: Ballistic protection mechanisms in personal armour. Journal of Materials Science, 36, 3137-3142 (2001). DOI: 10.1023/A:1017922000090

[2] Cwik T.: Survivability of structures to impact and blast loadings. Dstl/EPSRC progress report 1. Imperial Aeronautics Report E1 (2009).

[3] Cunniff P. M.: Dimensionless parameters for optimization of textile-based body armor systems. in ' $18^{\text {th }}$ International Symposium on Ballistics Conference, San Antonio, USA' 1303-1310 (1999).

[4] Grujicic M., Arakere G., He T., Bell W. C., Glomski P. S., Cheeseman B. A.: Multi-scale ballistic material modeling of cross-plied compliant composites. Composites Part B: Engineering, 40, 468-482 (2009). DOI: $10.1016 /$ j.compositesb.2009.02.002

[5] Iannucci L., Pope D., Dalzell M.: A constitutive model for Dyneema UD. in ' $17^{\text {th }}$ International Conference on Composite Materials, Edinburgh, United Kingdom' p.10 (2009).

[6] Iannucci L., Willows M.: An energy based damage mechanics approach to modelling impact onto woven composite materials - Part I: Numerical models. Composites Part A: Applied Science and Manufacturing, 37, 2041-2056 (2006).

DOI: 10.1016/j.compositesa.2005.12.013

[7] Greenhalgh E. S, Bloodworth V.: Fractographic assessment of dyneema composites under ballistic impact. Imperial Aeronautics Report, TR09/22/Cv1, (2009).

[8] Huang C., Chen S., Duan Z.: Experimental study on dynamic properties of high strength fiber clusters. Materials Science Forum, 475-479, 1045-1050 (2005). DOI: 10.4028/www.scientific.net/MSF.475-479.1045

[9] Farsi B., Dooraki J. A., Nemes M., Bolduc M.: Study of parameters affecting the strength of yarns. Journal de Physique IV: Proceedings, 134, 1183-1188 (2006). DOI: 10.1051/.jp4:2006134180

[10] Laffan M. J., Pinho S. T., Robinson P., Iannucci L.: Measurement of the in situ ply fracture toughness associated with mode I fibre tensile failure in FRP. Part I: Data reduction. Composites Science and Technology, 70, 606-613 (2010). DOI: $10.1016 /$ j.compscitech.2009.12.016

[11] Peus T., Smets E. A. M., Govaert L. E.: Strain rate and temperature effects on energy absorption of polyethylene fibres and composites. Applied Composite Materials, 1, 35-54 (1994). DOI: $10.1007 / \mathrm{BF} 00567210$ 
[12] Brooks N. W. J., Duckett R. A., Ward I. M.: Temperature and strain-rate dependence of yield stress of polyethylene. Journal of Polymer Science Part B: Polymer Physics, 36, 2177-2189 (1998).

DOI: 10.1002/(SICI)1099-0488(19980915)36:12<2177 $\because$ AID-POLB15>3.0.CO;2-X

[13] Govaert L. E., Peijs T.: Tensile strength and work of fracture of oriented polyethylene fibre. Polymer, 36, 4425-4431 (1995).

DOI: 10.1016/0032-3861(95)96848-3

[14] Hinman M. B., Jones J. A., Lewis R. V.: Synthetic spider silk: A modular fiber. Trends in Biotechnology, 18, 374-379 (2000).

DOI: $10.1016 / \mathrm{S} 0167-7799(00) 01481-5$
[15] Anderson C. E., Cox P. A., Johnson G. R., Maudlin P. J.: A constitutive formulation for anisotropic materials suitable for wave propagation computer programs - II. Computational Mechanics, 15, 201-203 (1994). DOI: $10.1007 / \mathrm{BF} 00375030$

[16] Raimondo L., Iannucci L., Robinson P., Pinho S. T.: A numerical material model for predicting the high velocity impact behaviour of polymer composites. Computational Methods in Applied Sciences, 10, 161177 (2008).

DOI: $\underline{10.1007 / 978-1-4020-8584-08}$ 